\title{
X-Ray Afterglows from Gamma-Ray Bursts
}

\author{
M. Tavani \\ Columbia Astrophysics Laboratory, Columbia University, New York, NY 10027 \\ IFCTR-CNR, via Bassini 15, Milano (Italy)
}

\begin{abstract}
We consider possible interpretations of the recently detected X-ray afterglow from the gamma-ray burst source GRB 970228. Cosmological and Galactic models of gamma-ray bursts predict different flux and spectral evolution of X-ray afterglows. We show that models based on adiabatic expansion of relativistic forward shocks require very efficient particle energization or post-burst re-acceleration during the expansion. Cooling neutron star models predict a very distinctive spectral and flux evolution that can be tested in current X-ray data.
\end{abstract}

Subject Headings: gamma rays: bursts, theory; X-rays: general

March 14, 1997

Submitted to the Astrophysical Journal Letters: March 18, 1997 


\section{Introduction}

The discovery by SAX of an X-ray afterglow from GRB 970228 (Costa et al.1997b,c, hereafter C97b,c) provides crucial information for the understanding of gamma-ray bursts (GRBs). This detection was possible because of a relatively rapid response in pointing to a GRB error box within a few hours. The results of two 'fast' Target of Opportunity (TOO) SAX pointings of GRB error boxes are currently available. An observation within 16 hrs of the $\sim 15 \operatorname{arcmin}^{2}$ error box of GRB 970111 (Hurley et al. 1997a) did not detect X-ray emission above a flux of $10^{-13} \mathrm{erg} \mathrm{cm}^{-2} \mathrm{~s}^{-1}$ (2-10 $\mathrm{keV}$ band) (Costa et al. 1997b). On the contrary, a pointing within 8 hours at the $\sim 6 \operatorname{arcmin}^{2}$ error box of GRB 970228 (Hurley et al. 1997b) resulted in the remarkable discovery of transient X-ray emission from a previously unknown source (C97c, Boller et al. 1997). The average X-ray flux level (2-10 keV band) of the first TOO pointing at the GRB 970228 error box is $\sim 3 \times 10^{-12} \mathrm{erg} \mathrm{cm}^{-2} \mathrm{~s}^{-1}$. A second observation carried out $\sim 3$ days later detected the same X-ray source at a substantially lower flux near $10^{-13} \mathrm{erg} \mathrm{cm}^{-2} \mathrm{~s}^{-1}$ (C97c). In this Letter we consider theoretical models that can reproduce the observed decay in X-ray flux by a factor of $\sim 30$ between the first (at $t \sim 8 \mathrm{hrs}$, with $t$ the elapsed time after the burst trigger) and second (at $t \sim 3.6$ days 86.6 hrs) SAX observations of GRB 970228. It is also interesting to note the absencef of a relatively strong X-ray source (at flux level near $10^{-12} \mathrm{erg} \mathrm{cm}^{-2} \mathrm{~s}^{-1}$ ) $\sim 16 \mathrm{hrs}$ after the GRB 970111 trigger. The peak intensities of GRB 970111 and GRB 970228 as detected by the GRBM instrument in the $60-600 \mathrm{keV}$ band are comparable within a factor of $\sim 2$ (C97a,b). We infer that temporal evolutions of X-ray afterglows from GRBs differ among bursts, and suggest that GRB models with no universal afterglow evolution are most likely.

\footnotetext{
1 Sources (a) and (b) detected by SAX in the WFC error box of GRB 970111 (Butler et al. 1997) are outside the refined error box of in 't Zand et al. (1997).
} 


\section{Cosmological models}

Coalescence of compact stars, failed supernovae, new-born spinars or special jet phenomena in distant galaxies can give rise to a rapidly expanding relativistic fireball dissipating its energy in internal and external shocks. To avoid pair opacity effects, these models require a large value of the relativistic bulk Lorentz factor $\Gamma \sim 10^{2}-10^{3}$ characterizing a fast-moving radiation front. The observed photon energies are upscattered by $\Gamma$ compared to the ones produced in the comoving frame. The observed spectral energy flux $F_{\nu}$ is $\Gamma^{5}$ times its comoving value, a factor of $\Gamma^{2}$ from the increasing solid angle from the apparent transverse dimension of the fireball $\left(r_{\perp}=c t \Gamma\right)$, and a factor of $\Gamma^{3}$ from relativistic beaming (Rees 1966). Adiabatic expansion applies to fireball emission occurring within a time $\sim 10^{3}-10^{4}$ times longer than the burst $T_{90}$ duration $\left(T_{90} \gtrsim 20 \mathrm{~s}\right.$ for GRB 970111 and GRB 970228, C97a,b). The initial burst may be produced by 'internal' or 'external' shocks for an initial value of $\Gamma=\Gamma_{o} \sim 10^{2}-10^{3}$ ( e.g., Mészáros \& Rees 1997, hereafter MR97). For an external shock, the deceleration radius is $r_{\text {dec }} \sim\left(10^{16} \mathrm{~cm}\right)\left(E_{51} / n_{o}\right)^{1 / 3} \Gamma_{3}^{-2 / 3}$, and the observed deceleration timescale $t_{d e c} \sim r_{d e c} /\left(c \Gamma^{2}\right)$, where $E_{51}$ is the initial fireball kinetic energy, and $n_{o}$ the particle number density in the surrounding medium (we use the convention $A_{x}=A /\left(10^{x}\right)$ ). We expect a temporal dependence of the type $\Gamma(t) \sim r^{-3 / 2} \propto t^{-3 / 8}$ and $r_{\|}=c t \Gamma^{2} \propto t^{1 / 4}$, with $r_{\|}$the longitudinal radius. If $I_{\nu^{\prime}}^{\prime}(t)$ is the (time-dependent) specific intensity in the shock comoving frame $\left(\nu^{\prime}=\nu / \Gamma\right)$, the observer frame flux at a frequency $\nu$ is then $F_{\nu} \propto r_{\perp}^{2} \Gamma(t)^{3} I_{\nu}^{\prime}(t) \sim t^{2} \Gamma(t)^{5} I_{\nu}^{\prime}(t)$ (MR97). The temporal evolution of $I_{\nu}^{\prime}(t)$ depends on the adopted model of emission.

The prompt burst high-energy behavior as observed in the $1 \mathrm{keV}-1 \mathrm{GeV}$ is consistent with synchrotron emission of impulsively accelerated particles (Tavani 1996a,b, hereafter T96a,b), possibly upscattered by inverse Compton (IC) effects (e.g., MR97). We adopt here the synchrotron model of prompt GRB emission from a fireball, and follow the evolution 
of the radiative shock as time progresses. IC is not expected to play an important role at the large values of $r_{\|}$implied by the X-ray afterglow of GRB 970228. A shock model of emission requires special conditions in order to have detectable X-rays 8-80 hrs after a GRB. Failed supernova (Woosley 1993), accretion-powered emission from massive black holes (e.g., Woosley 1996) and spindown-powered emission from newly born spinars (e.g., Usov 1992) may also produce delayed X-ray emission.

Let us assume that most of the emission of an expanding and progressively decelerating fireball is produced by the reverse shock, i.e., by the ejecta particles cooled by adiabatic expansion. The comoving intensity at $r_{\|}>r_{d e c}$ turns out to be $I_{\nu^{\prime}}^{\prime} \propto n_{e j}^{\prime} B^{\prime} \Delta r_{\|}^{\prime}$, where $n_{e j}^{\prime}$ is the ejecta radiating particle density, $B^{\prime}$ the magnetic field, and $\Delta r_{\|}^{\prime}=r_{\|} / \Gamma$. For adiabatic expansion, $n_{e j}^{\prime} \sim V^{\prime-1} \sim t^{-9 / 8}, B^{\prime} \sim V^{\prime-2 / 3} \sim t^{-3 / 4}$, and $\Delta r_{\|}^{\prime} \sim t^{5 / 8}$, where we assumed a frozen-in magnetic field with $V^{\prime}=4 \pi r_{\|}^{\prime 2} \Delta r_{\|}^{\prime}$ the comoving volume (MR97). We therefore obtain $I_{\nu^{\prime}}^{\prime} \sim t^{-5 / 4}$, and $F_{\nu} \sim t^{-9 / 8}$. The observed X-ray afterglow can be residual synchrotron emission of non-thermal particles accelerated by the prompt shock and adiabatically cooled. A non-thermal particle distribution function is assumed to be formed at the prompt GRB shock, as indicated by broad-band GRB spectra (T96a,b). This function is described by a relativistic Maxwellian low-energy component below the critical energy $\gamma_{*}$, and by a power-law component up to a maximum energy $\gamma_{\max }$ above $\gamma_{*}$. The detectability of an X-ray afterglow from an expanding fireball of this kind crucially depends on the synchrotron critical frequency $\nu_{*} \propto \Gamma \gamma_{*}^{2} B^{\prime}$ evolved at the radiating site. Since $\gamma_{*} \sim 1 / r \sim t^{-1 / 4}$, the critical frequency has a relatively fast decay, $\nu_{*}(t) \sim t^{-13 / 8}$. The observed X-ray flux $f_{x}(t)$ is the integral of $F_{\nu}(t)$ over a fixed energy band (say, 2-10 keV), and it turns out to be $f_{x}(t)=\xi_{x}(t) \nu_{*}(t) F_{\nu_{*}}(t)$, where $\xi_{x}(t)$ gives the fraction of total luminosity in the required energy range. We obtain $f_{x}(t)=\xi_{x}(t) t^{-21 / 8}$, i.e., a decay behavior substantially steeper than observed for GRB 970228 (but consistent with the lack of afterglow from GRB 970111). 
Shock emission from an adiabatically cooled fireball is predicted to be non-thermal. However, due to the strong temporal dependence of $\nu_{*}$, delayed X-ray emission from impulsively accelerated particles is possible only for very efficient energization. We can constrain the post-shock particle energization for no re-acceleration during adiabatic expansion at $r_{\|}>r_{d e c}$. The observed photon energy of the afterglow is assumed to be $E_{\text {max }} \sim \Gamma(t) \gamma_{\max }^{2}(t) B^{\prime}(t) \sim\left(t / t_{*}\right)^{-13 / 8}$. The decay timescale $t_{*}$ can be deduced from the time evolution of the peak energy of the $\nu F_{\nu}$ spectrum during a typical GRB, $\nu_{*} \sim(200 \mathrm{keV})\left(t / t_{*}\right)^{-13 / 8}$. A typical value is $t_{*} \simeq 3 \mathrm{~s}$. For no re-acceleration, we obtain

$$
\gamma_{\max }(0) / \gamma_{*}(0) \gtrsim 0.22\left(t / t_{*}\right)^{13 / 16}
$$

i.e., $\gamma_{\max }(0) / \gamma_{*}(0) \simeq 400$ for $t=8$ hrs. A substantial fraction of the shock luminosity is required to be emitted in X-rays even for values of $\nu_{*}$ substantially smaller than $h \nu_{*} \sim 1 \mathrm{keV}$. If we adopt $F_{\nu} \sim \nu^{-1}$ above $\nu_{*}$ (marginally consistent with the OSSE observation of GRB 970228 at $t=0.5 \mathrm{hrs}$, Matz et al. 1997), we expect the fraction $\xi_{x}$ to be constant during fireball evolution as long as Eq. 1 is satisfied. A steeper $F_{\nu}$ above $\nu_{*}$ would result in an X-ray flux steeper than $f_{x}(t) \propto t^{-21 / 8}$. In this model, synchrotron emission peaks in the optical range at $t / t_{*} \sim 1.8 \times 10^{3}$. However, due to the steep decrease of the bolometric luminosity, the delayed unabsorbed optical emission from pure synchrotron is too faint to be detectable.

An alternative model of fireball expansion relies on the emission by newly shocked material of the surrounding medium. In this case, the density of the radiating particles of the comoving frame is $n^{\prime}=\Gamma n_{o}$. In the absence of Rayleigh-Taylor instabilities at the contact discontinuity, the forward shock fluid does not mix with that of the reverse shock. The magnetic field can be deduced from equipartition arguments, $B^{\prime} \simeq \lambda_{B}^{1 / 2}\left(8 \pi n_{o} \Gamma \gamma_{*} m_{p} c^{2}\right)^{1 / 2}$, with $\lambda_{B}$ the efficiency of turbulent generation of magnetic field energy, and $m_{p}$ the proton's mass. It is interesting that in this case, $I_{\nu^{\prime}}^{\prime} \sim t^{-5 / 8}, F_{\nu} \sim t^{0}$, and $\nu_{*} \sim t^{-3 / 2}$ (e.g., MR97). 
We therefore deduce the decay behavior of the bolometric synchrotron emission in the 2-10 keV band as $f_{x}(t)=\xi_{x}(t) t^{-3 / 2}$. For a relatively constant $\xi_{x}(t)$, this function is marginally consistent with the observed afterglow of GRB 970228. As for the previous model, we can obtain the conditions on the acceleration mechanism that make possible delayed X-ray emission. If the local $\gamma_{*} \sim \Gamma$, and $\gamma_{\max } \sim \alpha^{\prime} \Gamma$, with $\alpha^{\prime} \gtrsim 1$, we can translate the condition on the observed photon energy as a requirement on initial values of $\gamma_{*}$ and $\gamma_{\max }$. For $E_{c}=10 \mathrm{keV}$ and $t=8 \mathrm{hrs}$, we obtain $\Gamma_{o}^{4} \sim 4.7 \times 10^{18}$, i.e, $\Gamma_{o} \simeq 4.6 \times 10^{4}$. More generally, we obtain for no re-acceleration

$$
\gamma_{\max }(0)^{2} \gamma_{*}(0)^{1 / 2} \simeq 4.7 \times 10^{15} n_{o}^{-1 / 2} \Gamma_{o, 2}^{-3 / 2} \lambda_{B}^{-1 / 2}(t / 8 \mathrm{hrs})^{3 / 2}
$$

We deduce that a forward shock may be consistent with the observed afterglow of GRB 970228, but only if the particle acceleration process of the forward shock material is very efficient and/or $\Gamma_{o}$ is very large. Fig. 1 show the results of the calculated flux in the X-ray and optical $\mathrm{R}$ bands for two different choices of the power-law index of the post-shock particle distribution function corresponding to $F_{\nu} \sim \nu^{-1}$ and $F_{\nu} \sim \nu^{-3 / 2}$, respectively. From Fig. 1 we deduce that in the absence of additional acceleration, the predicted X-ray flux does not agree with observations of GRB 970228 if $t_{*} \lesssim 100 \mathrm{~s}$. The resulting optical transient emission in the absence of absorption effects is shown in Fig. 1 (dot-dashed curves). The initial ratio of optical to X-ray flux is $\lesssim 10^{-5}$, implying an optical transient of R-magnitude $m_{R} \sim 18.4$. The transient evolves as $t^{-3 / 2}$ up to $t / t_{*} \sim 3 \times 10^{3}$ corresponding to the time when $\nu_{*}$ sweeps the optical band. For later times, the evolution of the optical flux becomes steeper. Detectable delayed radio emission may also be expected in this class of models (Paczyński \& Rhoads 1993).

If particle re-acceleration occurs during the adiabatic expansion phase, the constraints on $\gamma_{\max }(t)$ and $\gamma_{*}(t)$ become less stringent. Turbulent mixing of the reverse and forward shock fronts may favor efficient particle energization. In this case, Eq. 2 may not apply and 
a milder constraint for $\gamma_{\max }(t)$ and $\gamma_{*}(t)$ can be required. The evolution of both the X-ray and optical fluxes can be less steep than calculated here.

Models based on relativistic jets from failed supernovae (Woosley 1993) are also based on forward shock emission. Depending on the density of the surrouding medium, dissipation by synchrotron (or bremsstrahlung) emission can result in different values of the decay constant $t_{*}$ compared to models considered above. However, the qualitative features of the X-ray evolution should follow the trend given above. We can deduce an interesting constraint on the 'spinar' model for GRBs. A newly-born strongly magnetized neutron star can spin down very rapidly and make possible an initial fireball and subsequent non-thermal 'pulsar-like' emission (e.g., Usov 1992). In order to explain the required energy near $10^{52} \mathrm{erg} \mathrm{s}^{-1}$, this model assumes extreme values of the surface magnetic field $\sim 10^{15} \mathrm{G}$ and initial spin period $P \lesssim 10^{-3} \mathrm{~s}$. The spindown energy varies with time as $\sim\left(t / t_{s d}\right)^{-2}$, with

$t_{s d}$ the spindown timescale, $t_{s d} \sim \dot{P} / P \sim 100$ s. A fraction of the total spindown luminosity can be emitted as high-energy radiation. At $t=8 \mathrm{hrs}$, the emitted luminosity is decreased by a factor of $\sim 10^{-5}$ compared to the initial one. At $t=85 \mathrm{hrs}$, the decrease factor is $\sim 10^{-7}$. Therefore, this model predicts a luminosity evolution in disagreement with the observations of GRB 970228.

\section{Galactic models}

At a distance $d=d_{100} 100 \mathrm{kpc}$, an observed X-ray flux $F_{x}=F_{-12} 10^{-12} \mathrm{erg} \mathrm{cm}^{-2} \mathrm{~s}^{-1}$ corresponds to an isotropically radiated luminosity, $L_{x} \simeq 10^{36} F_{-12} d_{100}^{2} \mathrm{erg} \mathrm{s}^{-1}$. This sub-Eddington luminosity for a solar mass compact star can be produced in different ways. A sudden internal explosive event from a neutron star might cause matter liftoff and substantial release of energy in the external crust. Neutron stars in an extended Galactic halo require explosion energies of order of $10^{41}-10^{42}$ ergs. A super-Eddington luminosity 
leads to matter liftoff from the surface, possibly followed by matter fallback onto the surface of the neutron star. Let us consider first the case of internal energy release and cooling with no contribution from subsequent accretion. The thermal response of a neutron star crust depends on the depth at which most of the residual burst energy is liberated. For deep energy deposition, the timescale for conduction-driven cooling might be of orders of years or more. On the other hand, energy deposition in shallow layers of the external crust may lead to cooling timescales of order of $10-10^{4} \mathrm{~s}$. This can be seen by considering the initial photon diffusion time of the surface layers of a neutron star, $\tau_{d i f f} \sim 3 \kappa \rho x^{2} u_{t h} /\left(c u_{\gamma}\right)$, where $\kappa$ is the radiative opacity, $\rho$ the mass density, $x$ the depth (in $\mathrm{cm}$ ), $u_{t h}$ the thermal energy, $c$ the speed of light, and $u_{\gamma}$ the radiation energy density (e.g., Eichler \& Cheng 1989). For ${ }^{56} \mathrm{Fe}$ matter, we obtain $\tau_{\text {diff }} \simeq(30 \mathrm{~s})(x / 100)^{2}\left(T_{8} / 0.3\right)^{-5.5} \rho_{5}^{2.4}$. Thus a post-burst neutron star surface radiating near the Eddington limit can have $T_{8} \sim 0.3$ and $\tau_{\text {diff }} \sim 30 \mathrm{~s}$. Solutions of the conductive heat flow equation for vanishing depths depend on how the heat conductivity and heat capacity vary as a function of depth. This dependence is poorly known and we consider here the general form of the solution $T(t)=T_{o}\left(t / \tau_{c}\right)^{-1+\zeta}$, where $\zeta$ depends on the functional dependence of heat conductivity and capacity on depth.

We can then assume that the post-burst bolometric flux from a neutron star surface as detected by a distant observer depends on time as $L\left(t^{\prime}\right)=L_{o} t^{\prime-\alpha}$. This implies an effective surface temperature dependence $T\left(t^{\prime}\right)=T_{o} t^{\prime-\alpha / 4}$, where $t^{\prime}=t / \tau_{c}$ and $T_{o} \propto L_{o}^{1 / 4}\left(1-R_{\mathrm{Sch}} / R_{*}\right)^{-1 / 4}$, with $R_{*}$ and $R_{\mathrm{Sch}}$ the neutron star and Schwarzschild radii, respectively. For an initial luminosity radiated by the whole star near the Eddington limit $L_{E}=L_{38} 10^{38} \mathrm{erg} \mathrm{s}^{-1}$, we deduce an initial temperature, $T_{o} \simeq 2 \times 10^{7} L_{38}^{1 / 4} \mathrm{~K}$. It is interesting that for a decay timescale $\tau_{c} \sim 100 \mathrm{~s}$ and exponent $\alpha=3 / 2$, the temperature of a cooling neutron star surface would be in an optimal range for detection by X-ray instruments $\sim 1$ day after the burst. Fig. 2 shows the calculated energy flux in the $2-10 \mathrm{keV}$ energy band in units of the initial bolometric flux emitted by a cooling neutron star decaying as 
$f_{x}\left(t^{\prime}\right)=f_{o} t^{\prime-3 / 2}$, where $f_{o}=L_{o} /\left(4 \pi d^{2}\right)$. We consider two models characterized by different initial temperatures, $T_{o}=2 \times 10^{7} \mathrm{~K}$ and $T_{o}=3 \times 10^{7} \mathrm{~K}$, respectively. We notice that the X-ray fluxes detected approximately 8 and 85 hours following GRB 970228 qualitatively agree with expectations based on this simple model. An interpretation in terms of thermal afterglow implies $\zeta \simeq 5 / 8$, a value that can be used for a detailed modelling of the thermal response of a neutron star.

The cooling neutron star model predicts a substantial softening of the spectrum. Fig. 2 also shows the calculated evolution of the hardness $H$ defined as the ratio of photon fluxes in the $3-6 \mathrm{keV}$ and $2-3 \mathrm{keV}$ bands. The hardness ratio is predicted to vary by a factor $\sim 2$ within the first SAX TOO observation of GRB 970228. An even more drastic variation of the hardness dropping below 0.1 is predicted for later times, e.g., during the second SAX TOO observation. No detectable optical emission is expected in this case from pure neutron star cooling.

Other ways of producing X-ray emission can be considered. If the bulk of GRB emission occurs outside but not too far from a neutron star, photon irradiation and particle precipitation can further heat its surface. The energy deposition from above can lead to a thermal relaxation qualitatively similar to that of a cooling neutron star surface heated from below. However, the detailed heat transport may be different than in the previous case. Furthermore, fallback material from the burst explosion can settle in a disk or spherical inflow onto the neutron star surface. The X-ray spectrum in this case is predicted to be a combination of blackbody and power-law components as observed from several low-luminosity accreting neutron stars (White, Nagase \& Parmar 1995). X-ray pulsations might be detectable in case of emission from a strongly magnetized neutron star. The ratio of optical to X-ray luminosity is expected to be $10^{-2}-10^{-3}$, i.e., similar to faint accreting compact sources (e.g., White et al. 1995). 


\section{Discussion and conclusions}

We showed that the detection of an X-ray afterglow from a GRB strongly constrains theoretical models. Table 1 summarizes the main properties of the models considered here. Cosmological models based on adiabatic evolution of a synchrotron radiating shock front are the most constrained. The calculated emission in models of adiabatically expanding reverse shocks does not agree with observations of GRB 970228. Forward shock models might be compatible with observations only for very efficient particle acceleration, large decay timescale $t_{*} \gtrsim 100 \mathrm{~s}$ or re-acceleration during expansion. An optical transient of initial magnitude $m_{R} \sim 18-19$ lasting a few hours after the GRB is expected in this model. If $t_{*}=300 \mathrm{~s}$, we deduce from Fig. 1 an X-ray flux in qualitative agreement with the observations of GRB 970228 and an optical magnitude at $t / t_{*} \sim 100$ equal to $m_{R} \sim 22.7$, i.e., slightly higher than the limiting magnitude $\left(m_{R} \simeq 22\right)$ of the observations at $t=15.3 \mathrm{hr}$ reported by Guarnieri et al. (1997). Consistency with the optical transient possibly associated with GRB 970228 (Groot et al. 1997) requires $t_{*} \gtrsim 300 \mathrm{~s}$. No radio transient source above $\sim 1 \mathrm{mJy}$ has been reported in searches at $5 \mathrm{GHz}$ of the refined error boxes of GRB 970111 and GRB 970228 (Frail et al. 1997, Galama et al. 1997). We also showed that Galactic models of post-burst emission from cooling neutron stars have definite predictions. Different flux and spectral evolution patterns of X-ray afterglows are expected as a function of initial surface temperature and cooling timescale.

Our analysis is important for the interpretation of follow-up X-ray observations of GRB 970228. As shown in Fig. 2, in neutron star cooling models no detectable X-ray flux above $10^{-14} \mathrm{erg} \mathrm{cm}^{-2} \mathrm{~s}^{-1}$ from GRB 970228 is expected in the X-ray band for $t \gtrsim 100$ hrs. Analogously, cosmological models based on forward shocks with no re-acceleration, predict

an evolution of the X-ray flux $f_{x} \sim t^{-3 / 2}$ or steeper. Again, the predicted flux at $t \gtrsim 100$ hrs is below detectability with current instruments. A violation of these predictions would 
be remarkable, since it would point to a steady-state high-energy emission of the GRB counterpart.

We emphasize that X-ray afterglows from GRBs show different time evolutions. Within a given model, variations of physical properties (critical timescales, temperature, maximum value of particle energies in non-thermal models, etc.) can explain the difference between the afterglows from GRB 970111 and GRB 970228. A combined sequence of fast multiwavelength observations following GRBs can further constrain the emission parameters and their variations among different GRB sources.

The author thanks E. Costa, F. Frontera, M. Ruderman and M. Feroci for discussions. Research supported in parts by the NASA grant NAG5-2729. 


\section{References}

Band, D., et al., 1993, ApJ, 413, 281.

Boller, T. et al., 1997, IAU Circular no. 6580.

Butler, R.C., et al., 1997, IAU Circular no. 6539.

Costa, E., et al., 1997a, IAU Circular no. 6533.

Costa, E., et al., 1997b, IAU Circular no. 6572.

Costa, E., et al., 1997c, IAU Circular no. 6574.

Eichler, D. \& Cheng, A.F., 1989, ApJ, 336, 360.

Frail, D.A. et al., 1997, IAU Circular no. 6576.

Galama, R., et al., 1997, IAU Circular no. 6574.

Groot, P.J., et al., 1997, IAU Circular no. 6584.

Guarnieri, A., et al., 1997, IAU Circular no. 6582.

Hurley, K. et al., 1997a, IAU Circular no. 6571.

Hurley, K. et al., 1997b, IAU Circular no. 6578.

in 't Zand, J., et al., 1997, IAU Circular no. 6569.

Matz, S. et al., 1997, IAU Circular no. 6578.

Mészáros, P. \& Rees, M.J., 1997, ApJ, 476, 232.

Paczyński, B. \& Rhoads, J.E., 1993, ApJ, 418, L5.

Rees, M.J., 1966, Nature, 211, 468.

Tavani, M., 1996, PRL, 76, 3478.

Tavani, M., 1996b, ApJ, 466, 768.

Usov, V., 1992, Nature, 357, 472.

White, N.E., Nagase, F. \& Parmar, A.N. 1995, in X-Ray Binaries, eds. W.H.G. Lewin, J. van Paradijs \& E.P.J. van den Heuvel (Cambridge University Press), p. 1.

Woosley, S. E., 1993, ApJ, 405, 273.

Woosley, S. E., 1996, in Gamma-Ray Bursts, eds. C. Kouveliotou, M.F. Briggs, G.J. Fishman (AIP Conf. Proc. no. 384), p. 709. 


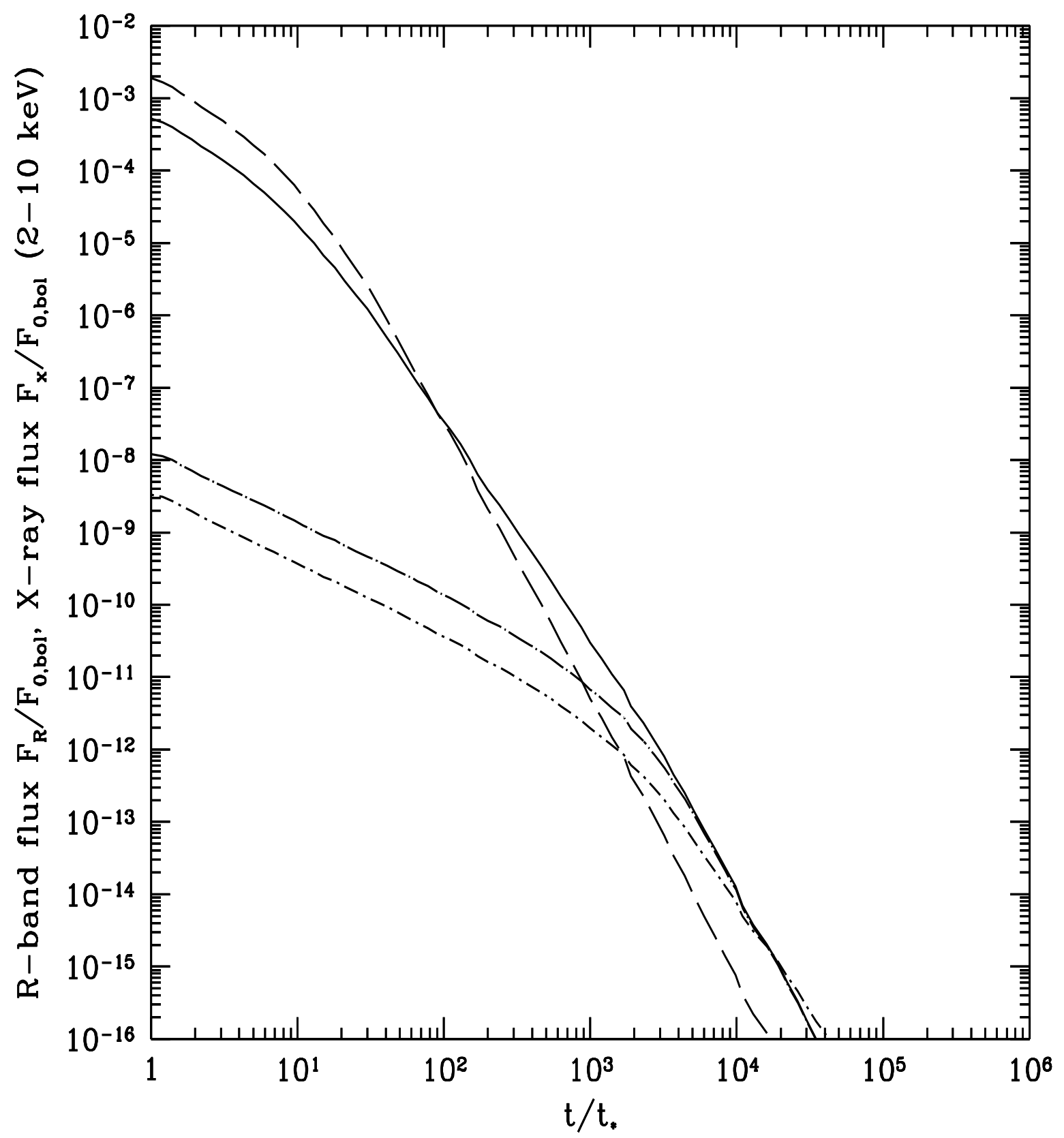

FIG. 1 - Calculated evolution of X-ray and optical (R-band) fluxes for an adiabatically expanding forward shock with no re-acceleration. The temporal axis scale is in units of $t_{*}$, with the synchrotron critical frequency evolving as $\nu_{*} \sim\left(t / t_{*}\right)^{-3 / 2}$. (Solid curve:) X-ray (2-10 keV) flux (in units of the initial bolometric flux) for a spectral form $F_{\nu} \sim \nu^{-1}$ above $\nu_{*}$; (long-dashed curve:) the same for $F_{\nu} \sim \nu^{-3 / 2}$ above $\nu_{*}$. (Long-dashed-dotted curve:) R-band flux (in units of the initial bolometric flux) for $F_{\nu} \sim \nu^{-1}$ above $\nu_{*}$; (short-dashed-dotted curve:) the same for $F_{\nu} \sim \nu^{-3 / 2}$ above $\nu_{*}$. 


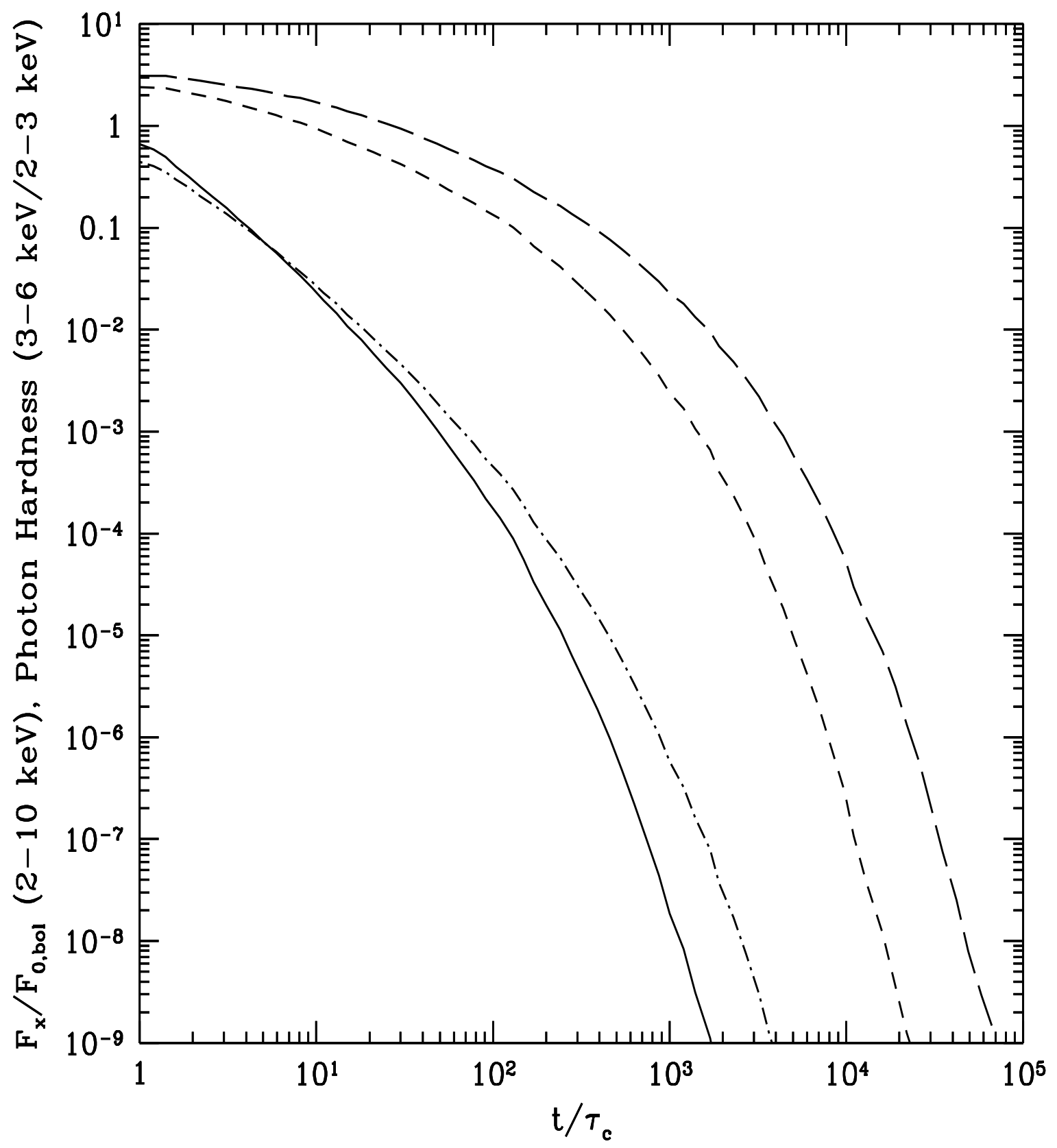

FIG. 2 - Evolution of X-ray flux and photon hardness as a function of $t / \tau_{c}$ for the cooling neutron star model. (Solid curve:) X-ray flux $(2-10 \mathrm{keV})$ in units of $F_{o, b o l}$ for an initial temperature $T_{o}=2 \times 10^{7} \mathrm{~K}$; (dashed-dotted curve:) the same for $T_{o}=3 \times 10^{7} \mathrm{~K}$. (Short-dashed curve:) photon hardness ratio for the channels $3-6$ and $2-3 \mathrm{keV}$ for $T_{o}=2 \times 10^{7} \mathrm{~K}$; (long-dashed curve:) photon hardness ratio for $T_{o}=3 \times 10^{7} \mathrm{~K}$. 
TABLE 1: Models for X-ray afterglows from GRBs

\begin{tabular}{lll}
\hline Model & $f_{x}(t)$ & Requirements \\
\hline Adiabatic fireball: reverse shock & $\xi_{x} t^{-21 / 8}$ & $\xi_{x} \sim$ const.; $\gamma_{\max }(0) / \gamma_{*}(0) \gtrsim 400$ \\
Adiabatic fireball: forward shock & $\xi_{x} t^{-3 / 2}$ & $\xi_{x} \sim$ const.; $\gamma_{\max }(0)^{2} \gamma_{*}(0)^{1 / 2} \gtrsim 4.7 \times 10^{15} n_{o}^{-1 / 2} \Gamma_{o, 2}^{-3 / 2}$ \\
Failed supernova & $\xi_{x} t^{-3 / 2}$ & Optically thin circumstellar medium \\
Spindown-powered emission & $t^{-2}$ & Strongly magnetized, rapidly rotating compact star \\
NS cooling & $t^{-1+\zeta}$ & Shallow energy deposition, $\zeta \sim 5 / 8$ \\
NS cooling with external irradiation & $t^{-1+\zeta^{\prime}}$ & Substantial reprocessing, $\zeta^{\prime} \sim 5 / 8$ \\
\hline
\end{tabular}

